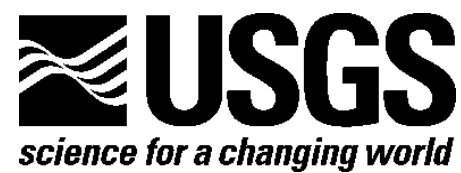

Prepared as part of the U.S. Geological Survey Priority Ecosystems Science Initiative

\title{
Joint Ecosystem Modeling (JEM) Ecological Model Documentation Volume 1: Estuarine Prey Fish Biomass Availability v1.0.0
}

By Stephanie S. Romañach, Craig Conzelmann, Adam Daugherty, Jerome J. Lorenz, Christina Hunnicutt, and Frank J. Mazzotti

Open-File Report 2011-1272

U.S. Department of the Interior

U.S. Geological Survey 


\section{U.S. Department of the Interior \\ KEN SALAZAR, Secretary}

\section{U.S. Geological Survey \\ Marcia K. McNutt, Director}

U.S. Geological Survey, Reston, Virginia 2011

For product and ordering information:

World Wide Web: http://www.usgs.gov/pubprod

Telephone: 1-888-ASK-USGS

For more information on the USGS-the Federal source for science about the Earth,

its natural and living resources, natural hazards, and the environment:

World Wide Web: http://www.usgs.gov

Telephone: 1-888-ASK-USGS

Suggested citation:

Romañach, S.S., Conzelmann, C., Daugherty, A., Lorenz, J.L., Hunnicutt, C., and Mazzotti, F.J. 2011, Joint Ecosystem Modeling (JEM) Ecological Model Documentation Volume 1: Estuarine Prey Fish Biomass Availability v1.0.0: U.S Geological Survey Open-File Report 2011-1272, 20 p.

Any use of trade, product, or firm names is for descriptive purposes only and does not imply endorsement by the U.S. Government.

Although this report is in the public domain, permission must be secured from the individual copyright owners to reproduce any copyrighted material contained within this report. 


\section{Acknowledgments}

We appreciate the input from many individuals from our partner agencies, organizations, and universities who participated in a workshop to help improve the model. Thanks is extended to Kevin Chartier for programming support. We appreciate the time spent by two anonymous reviewers whose comments resulted in a better product.

We are grateful the Advanced Applications Group at the National Wetlands Research Center for providing a platform (EverVIEW) for viewing and examining the model inputs and outputs. Many thanks to G. Ronnie Best and Greater Everglades Priority Ecosystems Science for funding to develop the software. 


\section{Contents}

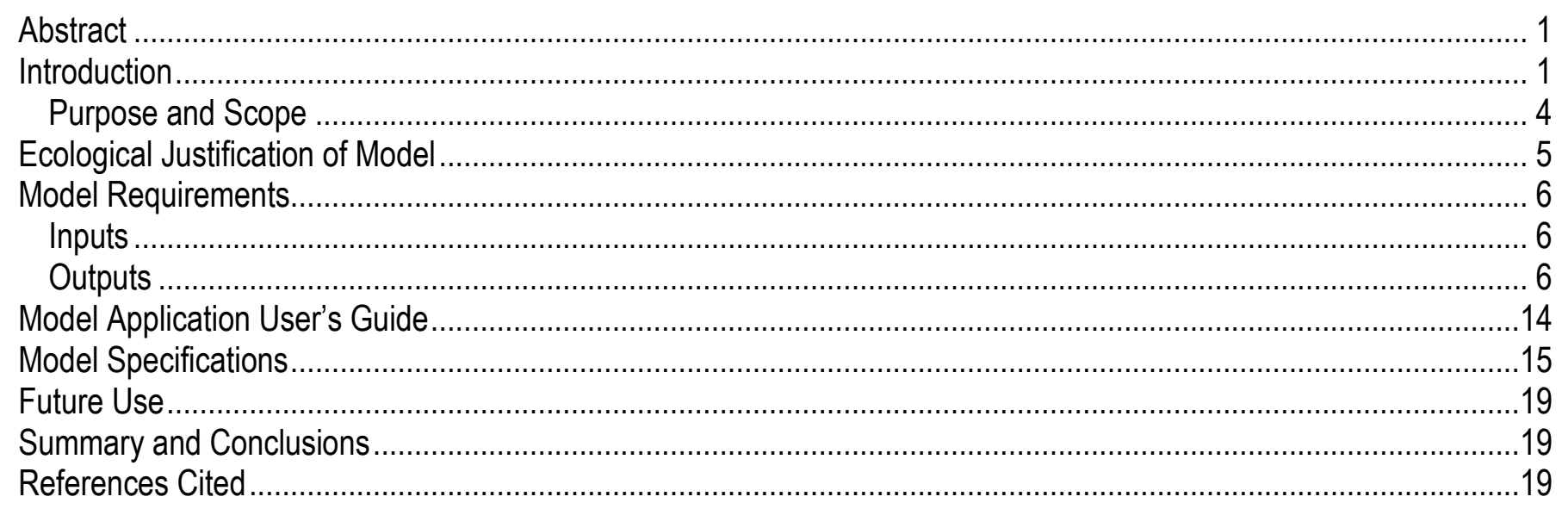

\section{Figures}

Figure 1. Schematic illustration of water flow in the Greater Everglades ............................................................. 1

Figure 2. Map showing location of Taylor Slough and northeastern Florida Bay................................................... 3

Figure 3. Map showing Estuarine Prey Fish Biomass Availability model output from the 90-day low salinity day count on December 29, 1999 ..................................................................................................

Figure 4. Map showing Estuarine Prey Fish Biomass Availability model output from the mean 300-day depth function on December 29, 1999................................................................................................. 8

Figure 5. Map showing Estuarine Prey Fish Biomass Availability model output from the 90-day depth standard deviation function on December 29, 1999 .................................................................................... 9

Figure 6. Map showing Estuarine Prey Fish Biomass Availability model output from the 60-day low depth day count function on December 29, 1999 ……................................................................................10

Figure 7. Map showing Estuarine Prey Fish Biomass Availability model output from the continuous high depth

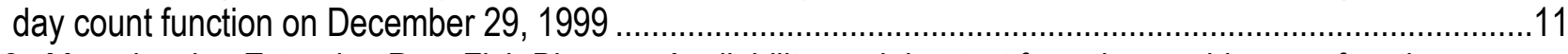

Figure 8. Map showing Estuarine Prey Fish Biomass Availability model output from the raw biomass function on December 29, 1999

Figure 9. Map showing Estuarine Prey Fish Biomass Availability model output from the biomass index showing change through time on December 27, 1997, 1998, and 1999.

\section{Tables}

Table 1. Outputs to be produced by the Estuarine Prey Fish Biomass Availability model.

Table 2. Variables in the output NetCDF files as they relate to inputs and outputs for the Estuarine Prey Fish Biomass Availability model. 


\title{
Joint Ecosystem Modeling (JEM) Ecological Model Documentation Volume 1:Estuarine Prey Fish Biomass Availability v1.0.0
}

\author{
By Stephanie S. Romañach¹, Craig Conzelmann², Adam Daugherty ${ }^{3}$, Jerome J. Lorenz ${ }^{4}$, Christina Hunnicutt², \\ and Frank J. Mazzotti ${ }^{3}$
}

\begin{abstract}
Estuarine fish serve as an important prey base in the Greater Everglades ecosystem for key fauna such as wading birds, crocodiles, alligators, and piscivorous fishes. Human-made changes to freshwater flow across the Greater Everglades have resulted in less freshwater flow into the fringing estuaries and coasts. These changes in freshwater input have altered salinity patterns and negatively affected primary production of the estuarine fish prey base. Planned restoration projects should affect salinity and water depth both spatially and temporally and result in an increase in appropriate water conditions in areas occupied by estuarine fish. To assist in restoration planning, an ecological model of estuarine prey fish biomass availability was developed as an evaluation tool to aid in the determination of acceptable ranges of salinity and water depth. Comparisons of model output to field data indicate that the model accurately predicts prey biomass in the estuarine regions of the model domain. This model can be used to compare alternative restoration plans and select those that provide suitable conditions.
\end{abstract}

\section{Introduction}

Development for human needs in South Florida led to a system of canals being constructed with the result of diverting fresh water away from much of the Greater Everglades ecosystem, including diverting freshwater flow away from estuaries and coasts (fig. 1). The Comprehensive Everglades Restoration Plan (CERP) presented to Congress in July 1999 (U.S. Army Corps of Engineers and South Florida Water Management District, 1999) recommends over 60 projects with the goal of restoring predevelopment water flow across the Greater Everglades. The restoration of predevelopment water flow should lead to more natural hydroperiods across the landscape.

\footnotetext{
${ }^{1}$ Southeastern Ecological Science Center, U.S. Geological Survey

${ }^{2}$ National Wetlands Research Center, U.S. Geological Survey

${ }^{3}$ Fort Lauderdale Research and Education Center, University of Florida

${ }^{4}$ Audubon of Florida
} 

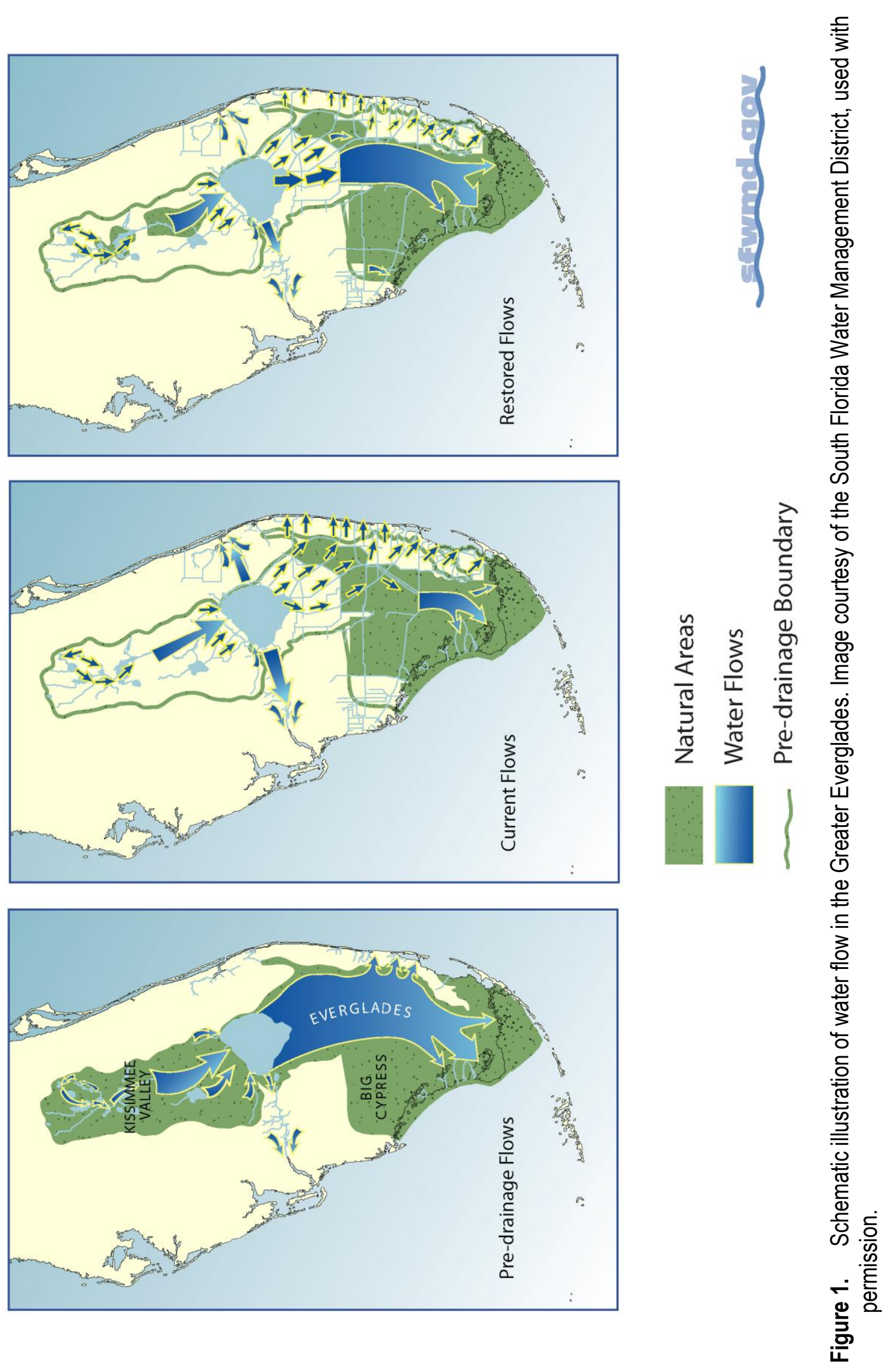


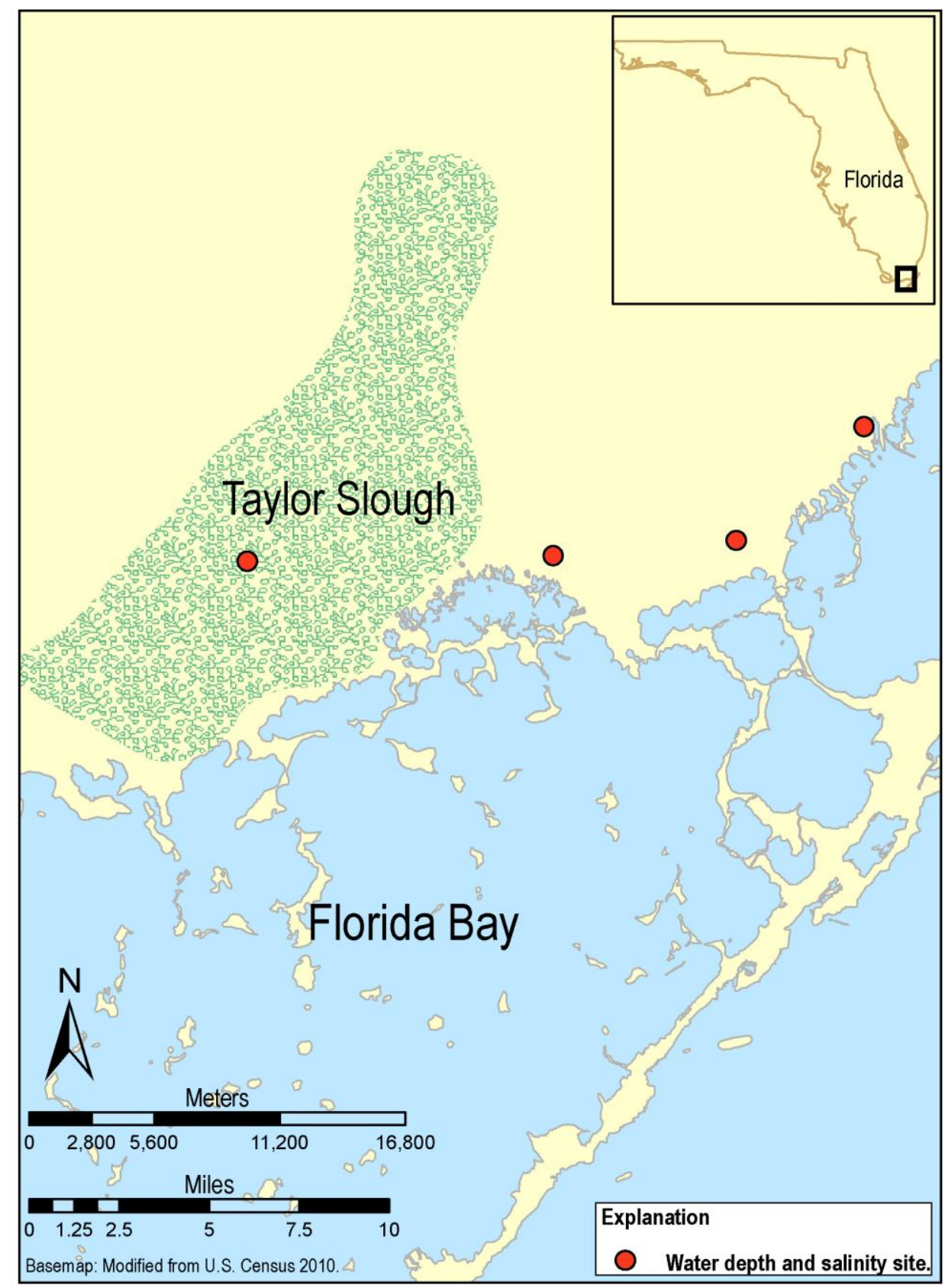

Figure 2. Map showing location of Taylor Slough and northeastern Florida Bay. Site descriptions are provided in the Ecological Justification of Model section.

Freshwater flow into the coastal areas provided conditions necessary for persistence of estuarine fauna and flora. After inland canals and water control structures were constructed, water delivery to areas such as Taylor Slough and northeastern Florida Bay (fig. 2) changed (Light and Dineen, 1994; Lorenz, 2000; McIvor and others, 1994). Florida Bay, for example, has experienced major changes in salinity, vegetation, flora, and fauna, causing the populations of many species to change (Bancroft and others, 1994; Loftus and Eklund, 1994). One of the major restoration goals for Florida Bay is to restore Taylor Slough as a primary source of freshwater for the eastern and central areas of the bay. Planned restoration projects will affect salinity and water depth spatially and temporally. 
Changes in salinity patterns have been shown to reduce primary production of fish through stresses caused by rapid and frequent fluctuations in salinity (Frezza and Lorenz, 2003; Montague and Ley, 1993; Ross and others, 2000). These changes alter the prey-base fish community to a state of lower secondary production (Lorenz, 1999; Lorenz and Serafy, 2006). The persistence of these fish is important in the Greater Everglades ecosystem as they are prey to many key fauna such as wading birds, crocodiles, alligators, and piscivorous fishes.

To assist in restoration planning, ecological models were developed by the U.S. Geological Survey (USGS) and others as an evaluation tool to help determine an acceptable range of conditions, such as for salinity and water depth, as they relate to the persistence of key fauna. These models can be used to select among alternative restoration plans that provide the most suitable conditions for a given species.

The model described herein helps contribute to USGS terrestrial, freshwater, and marine environments ecosystem science. The model helps users to understand the ecosystem and how various factors control prey fish availability and to predict future changes as Greater Everglades restoration progresses.

\section{Purpose and Scope}

This report describes the details of an Estuarine Prey Fish Biomass Model application (hereafter biomass model) using water depth and salinity inputs to predict estuarine prey fish biomass availability. The data used to create the water depth and salinity relations used as inputs to the model were published by Lorenz and others (1999).

Model results are given for the entire geographic domain of the water depth and salinity inputs, both of which are from the Tides and Inflows in the Mangroves of the Everglades (TIME) model (Wang and others, 2007); however, the biomass model has been validated only at the four sampling estuarine sampling sites (fig. 2). We caution users about extending their interpretation of model results into the freshwater region of the Greater Everglades, as this model is intended only to be meaningful for estuarine prey fish biomass.

The information presented in this report is divided into four major sections:

1. Ecological Justification of Model: Provides ecological background for model rules.

2. Model Requirements: Describes the required input data, what the outputs of the model are, and the rules that define the model. This section is intended to be used by subject matter experts and modelers to evaluate model rules, and by software engineers as a part of the analytical phase of software development.

3. Model Application User's Guide: Describes how to use the model application to generate model results. It is intended to be used by decision makers or anyone who wishes to generate model results.

4. Model Specifications: Provides information related to design of the model application, limitations of model application and data, formats for inputs and outputs, and extension and adaptation of the model. It is intended to provide coding details relevant to software developers. 


\section{Ecological Justification of Model}

The decision rules for this model were developed based on data collected by Lorenz (1999) as part of a study on roseate spoonbill ecology in Florida Bay. Water depth and salinity were measured from continuous data recorders at each of four sites in Florida Bay (fig. 2) over a 5-year period (see Lorenz, 1999, for details of data collection). The four sampling sites were within the primary foraging area of roseate spoonbill colonies. Sites were in dwarf mangrove habitat with a deep central creek, and were surrounded by shallow flats.

Thresholds were determined for physical variables. For water depth, 5 centimeters $(\mathrm{cm})$ was selected as this is the depth at which mud flats become exposed; $0 \mathrm{~cm}$ is too low because at this level the flats are completely dry (Lorenz, 1999). Salinity of 5 parts per thousand (ppt) was chosen because at greater salinities, submerged aquatic vegetation can be negatively affected, thereby having a negative impact on fishes that rely on it for food and habitat (Lorenz, 1999). Lorenz (2000) demonstrated that fish begin to evacuate the flats and take refuge in deeper habitats when water levels drop to a relative depth of about $12.5 \mathrm{~cm}$; however, more recent analyses indicate that a threshold of $13.1 \mathrm{~cm}$ is more accurate (J.J., Lorenz, Audubon of Florida, written commun. 2010).

Means and standard deviations for salinity and water level were calculated for the day on which each sample was collected and for the 30-, 60-, 90-, 120-, 180-, 240-, 300-, and 360-day periods prior to each sampling (Lorenz, 1999). Stepwise (forward and backward) regressions were performed for biomass using various measures for water depth and salinity (Lorenz, 1999).

The following variables contributed to explaining most of the variance in fish biomass: low salinity period (positive relationship), long-term variation in salinity (positive relationship), short-term variation in water levels (negative relationship), mean salinity (negative relationship), and long-term mean water levels (positive relationship). As such, the biomass regression (equation 1) is calculated as a function of water depth, 90-day low salinity day count, mean 300-day depth, 90-day depth standard deviation, 60-day low depth day count, and continuous high depth day count.

$1.01+$

$0.0013 \times($ number of days of last 90 that salinity was less than $5 \mathrm{ppt})+$

$0.037 \times($ mean depth of last 300 days, in $\mathrm{cm})+$

$(-0.041) \times($ standard deviation of depth for past 90 days, in $\mathrm{cm})+$

$(-0.02) \times($ current day depth, in $\mathrm{cm})+$

$(-0.01) \times($ number of days of last 60 that depth was less than $5 \mathrm{~cm})+$

$0.0013 \times$ (number of continuous days with depth greater than $13.1 \mathrm{~cm}$ )

Biomass predicted by the model significantly $(\mathrm{p}<0.0001)$ explained about a third of the variability $\left(\mathrm{r}^{2}=0.32\right)$ in the observed biomass. This degree of correlation between prediction and observation is considered to be reasonable for community data of this type (DeAngelis and others, 1997; Lorenz, 1999). 


\section{Model Requirements}

\section{Inputs}

Two input files are needed to generate model results. The first input is water depth, measured in meters (converted into centimeters by the model) and the second is salinity, measured in parts per thousand; both inputs are a series of time steps of maps, or geospatial grids. All maps (in both inputs) must have the exact same shape, scale, geographical location, and coordinate system for the model to work as formulated herein. Both inputs must be exactly 1 day, with no missing time steps between the first and last date (in other words, the inputs must be contiguous). There must be at least 300 days of input data for outputs to be generated.

Table 1. Outputs to be produced by the Estuarine Prey Fish Biomass Availability model.

\begin{tabular}{ll}
\hline \multicolumn{1}{c}{ Output } & \multicolumn{1}{c}{ Inputs } \\
\hline 90-Day Low Salinity Day Count & Salinity \\
Mean 300-Day Depth & Water Depth \\
90-Day Depth Standard Deviation & Water Depth \\
60-Day Low Depth Day Count & Water Depth \\
Continuous High Depth Day Count & Water Depth \\
Raw Biomass & Water Depth, 90-Day Low Salinity Day \\
& Count, Mean 300-Day Depth, 90-Day \\
& Depth Standard Deviation, 60-Day Low \\
& Depth Day Count, Continuous High Depth \\
& Day Count \\
Biomass Index & Raw Biomass \\
\hline
\end{tabular}

\section{Outputs}

A number of outputs are produced by the model (table 1). Some of these outputs are model results, whereas other outputs exist to verify model results and to examine the causes and factors that contributed to the results obtained. The output column of table 1 contains the name of the output to be produced. The input column lists the items required to produce the output; for example, 90-day low salinity day count is a function of the salinity data, and whenever the salinity data are changed, the 90-day low salinity day count also will change. All of these outputs will be produced either from all time steps of its inputs or from a subset of time steps of its inputs. All of these outputs are maps that have the same exact shape, scale, geographic location, and coordinate system as the input layers. 


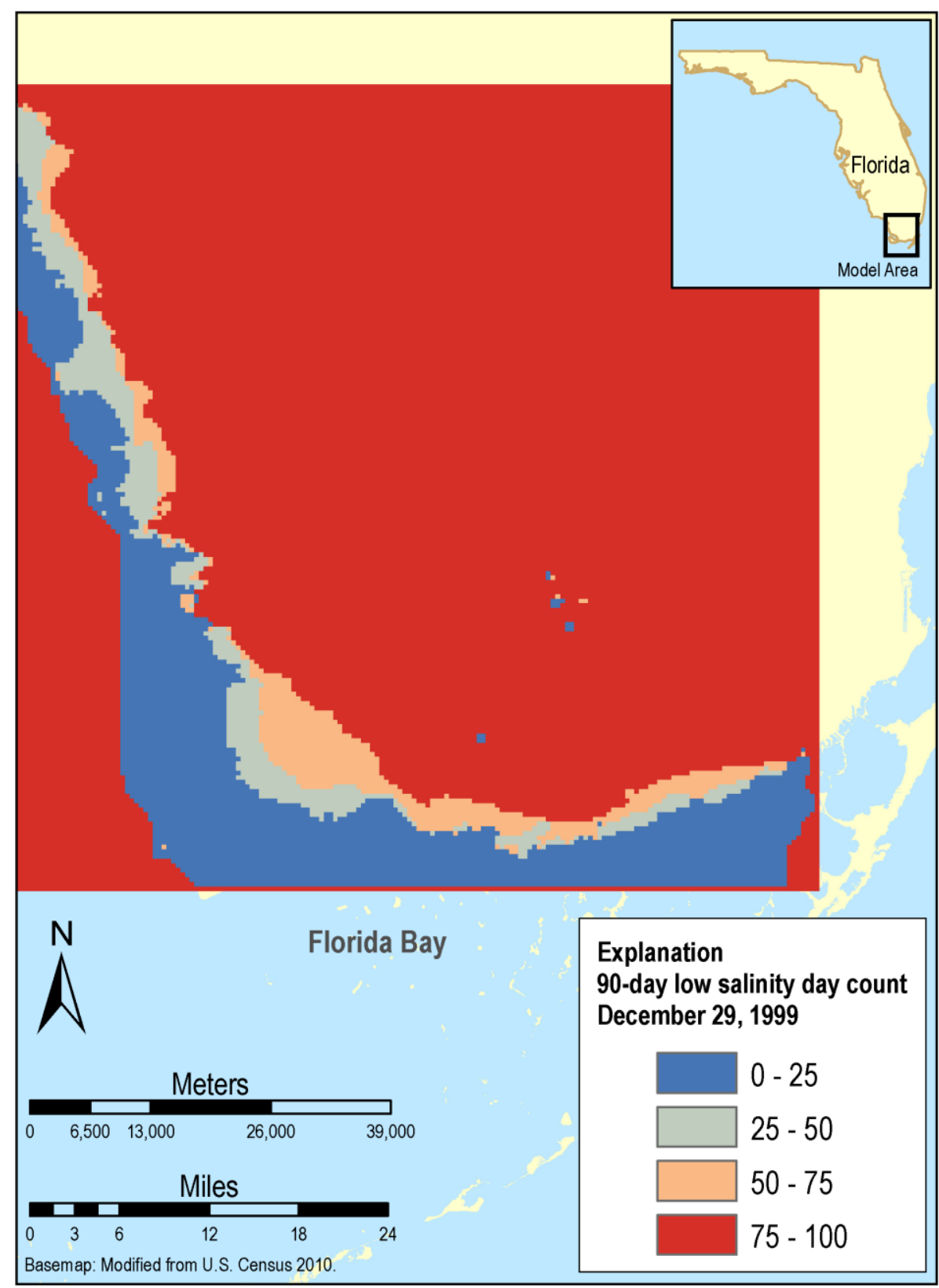

Figure 3. Map showing Estuarine Prey Fish Biomass Availability model output from the 90-day low salinity day count on December 29, 1999. Red indicates greater number of days and blue indicates lower count of days.

90-day low salinity day count-This count is a function of the salinity input. A single map is produced for each day of salinity input after the 89th day of the salinity time series (fig. 3). This output contains cells whose values are a count of the total number of days within the last 90 days (including the day for which the output is being generated), during which the cell had a salinity value of fewer than 5 ppt. 


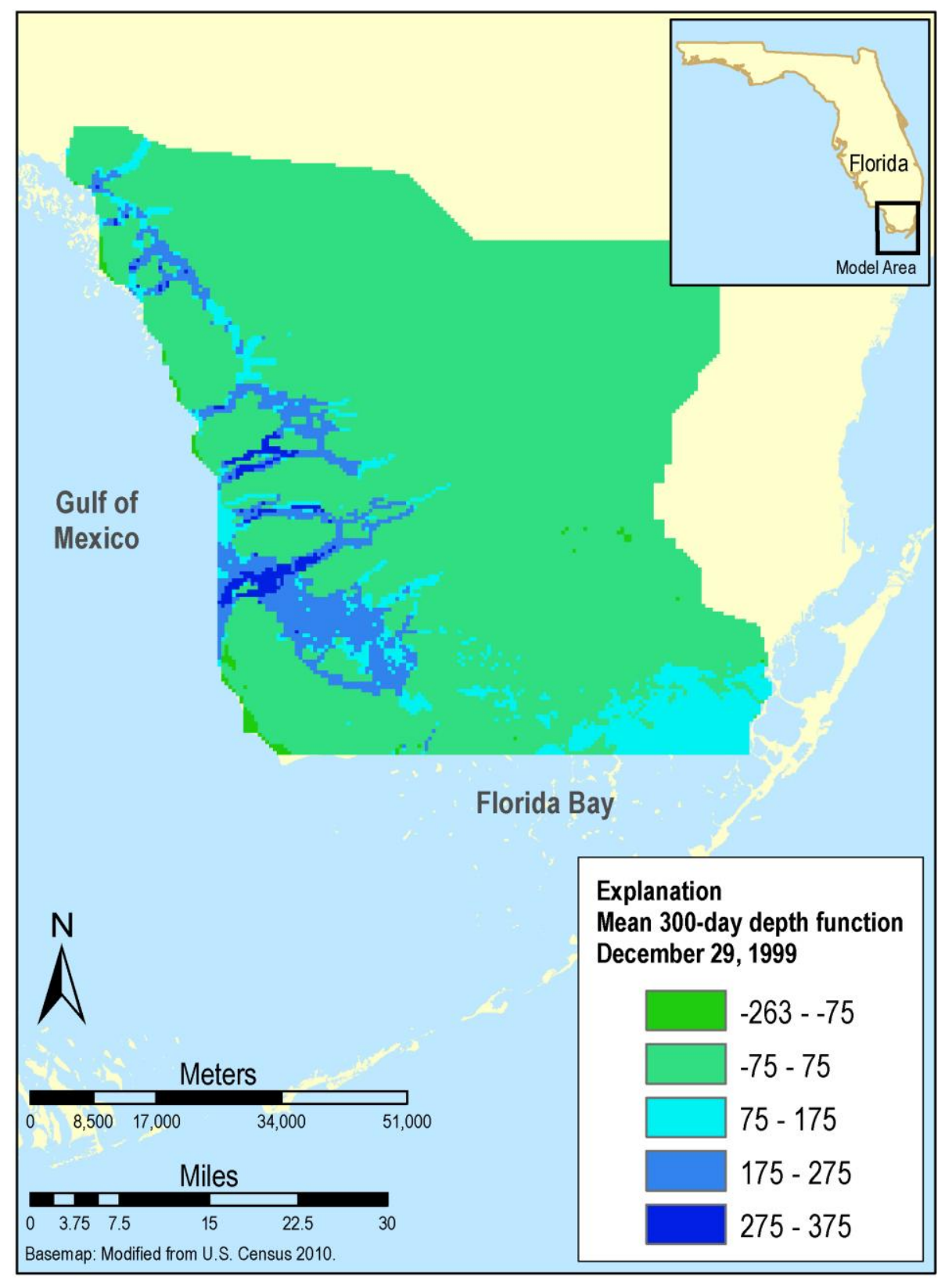

Figure 4. Map showing Estuarine Prey Fish Biomass Availability model output from the mean 300-day depth function on December 29, 1999. Blue indicates greater water depth than green.

Mean 300-day depth-This measure is a function of the water depth input. A single map is produced for each day of the water depth input after the 299th day of the water depth time series (fig. 4). This output contains cells whose values are the arithmetic mean of the values in that cell in the water depth time series for the last 300 days (including the day for which the output is being generated). 


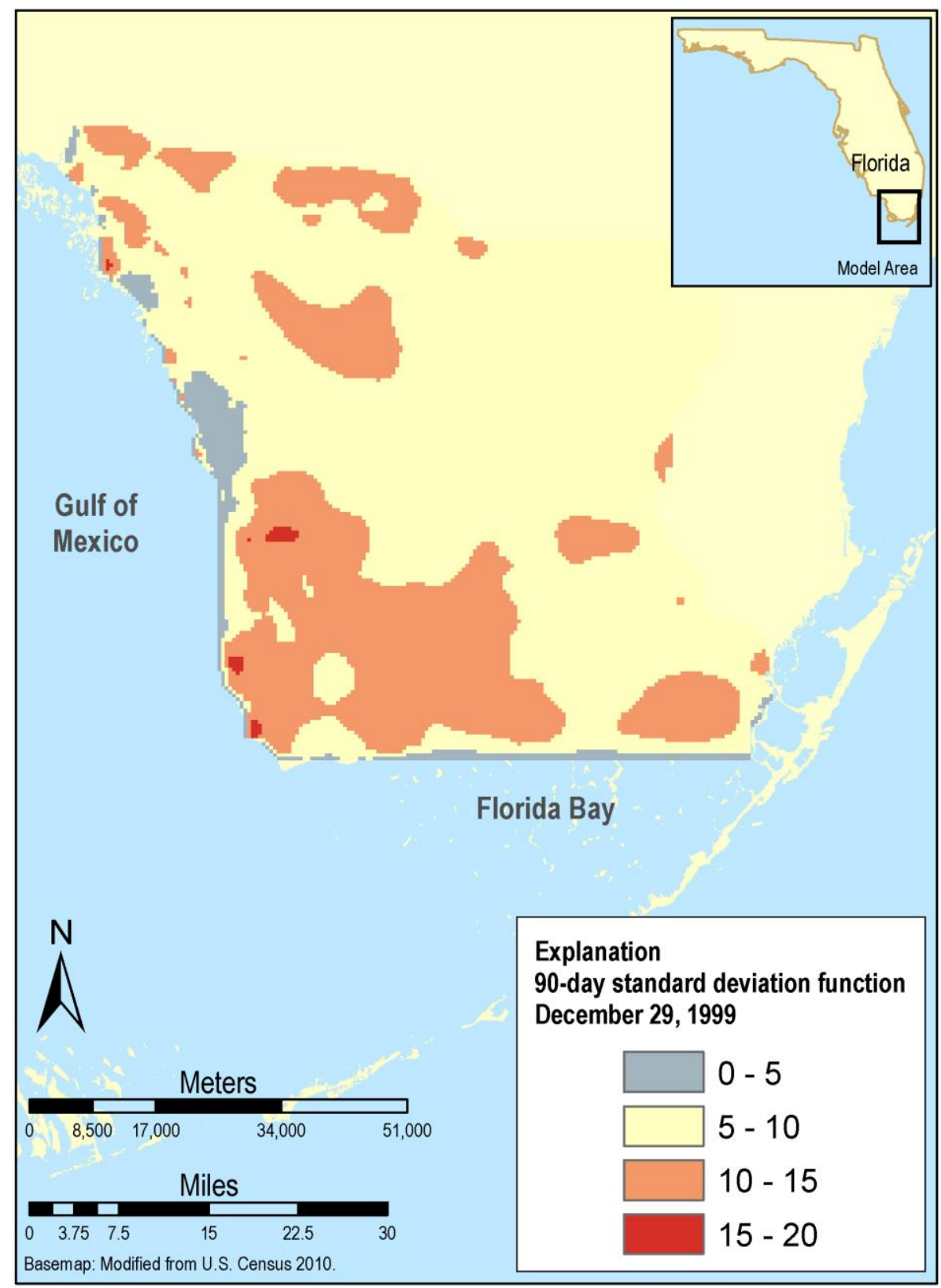

Figure 5. Map showing Estuarine Prey Fish Biomass Availability model output from the 90-day depth standard deviation function on December 29, 1999. Red indicates highest standard deviation.

90-day depth standard deviation-This calculation is a function of the water depth input. A single map is produced for each day of water depth input after the 89th day of the water depth time series (fig. 5). This output contains cells whose values are the standard deviation of all values in that cell in the water depth time series for the last 90 days (including the day for which output is being generated). 


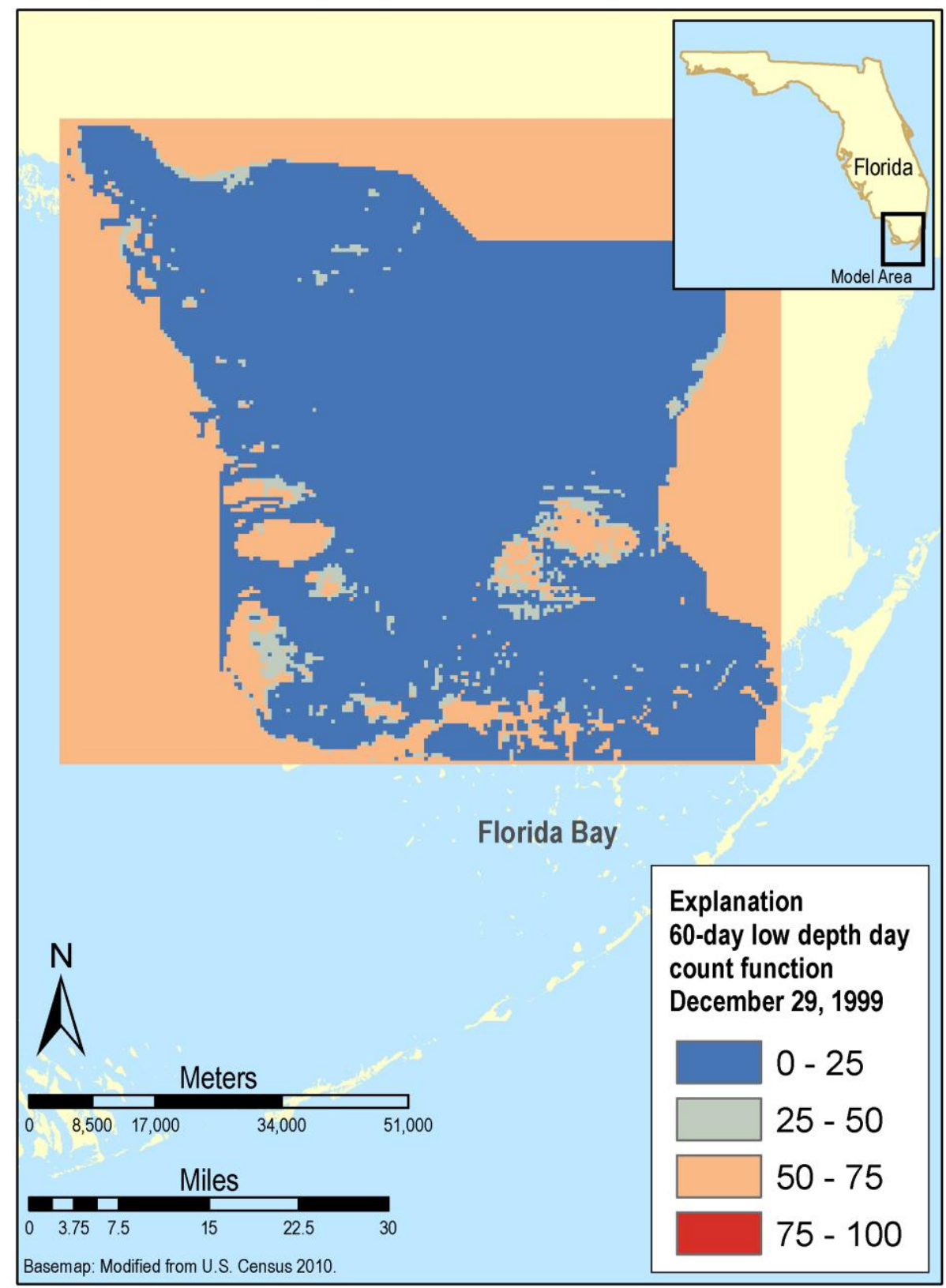

Figure 6. Map showing Estuarine Prey Fish Biomass Availability model output from the 60-day low depth day count function on December 29, 1999. Red indicates greater day count and blue indicates lower day count.

60-day low depth day count-This measure is a function of the water depth input. A single map is produced for each day of the water depth input after the 59th day of the water depth time series (fig. 6). This output contains cells whose values are a count of the total number of days in the last 60 days (including the day for which the output is being generated) during which the cell had a water depth of fewer than $5 \mathrm{~cm}$. 


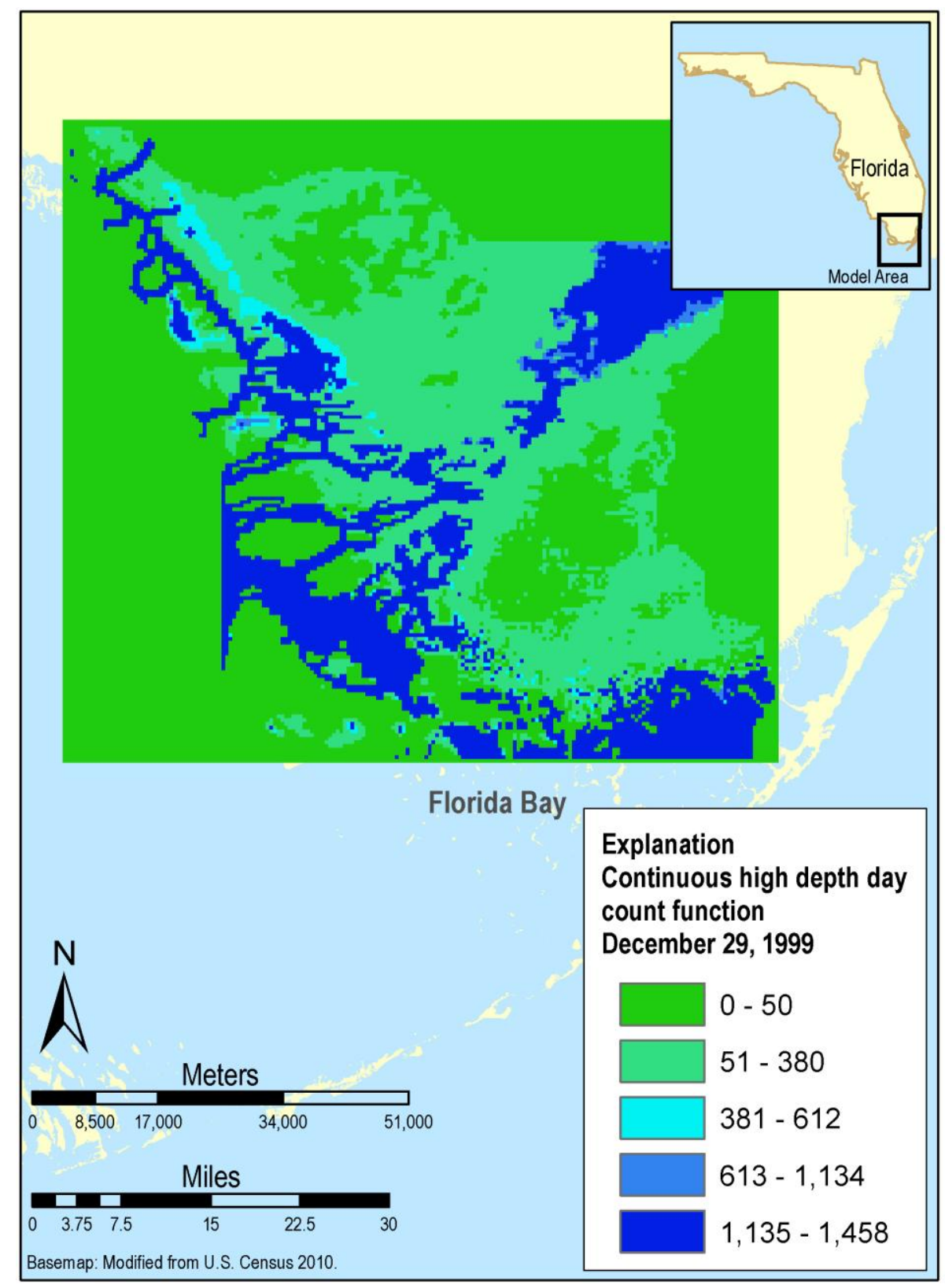

Figure 7. Map showing Estuarine Prey Fish Biomass Availability model output from the continuous high depth day count function on December 29, 1999. Blue indicates greater day count and green indicates lower day count.

Continuous high depth day count-This count is a function of water depth input. A single map is produced for each day of water depth input (fig. 7). This output contains cells whose values are a count of the total number of days during which the cell continuously had a water depth greater than $13.1 \mathrm{~cm}$. Restated, either the output for the first day should be zero, and output for every day thereafter should have a value of zero if the cell has a water depth less than or equal to $13.1 \mathrm{~cm}$ on the previous day, or the cell should have a value equal to the continuous high depth day count output for the previous day plus one if the cell for the previous day has a water depth greater than $13.1 \mathrm{~cm}$. 


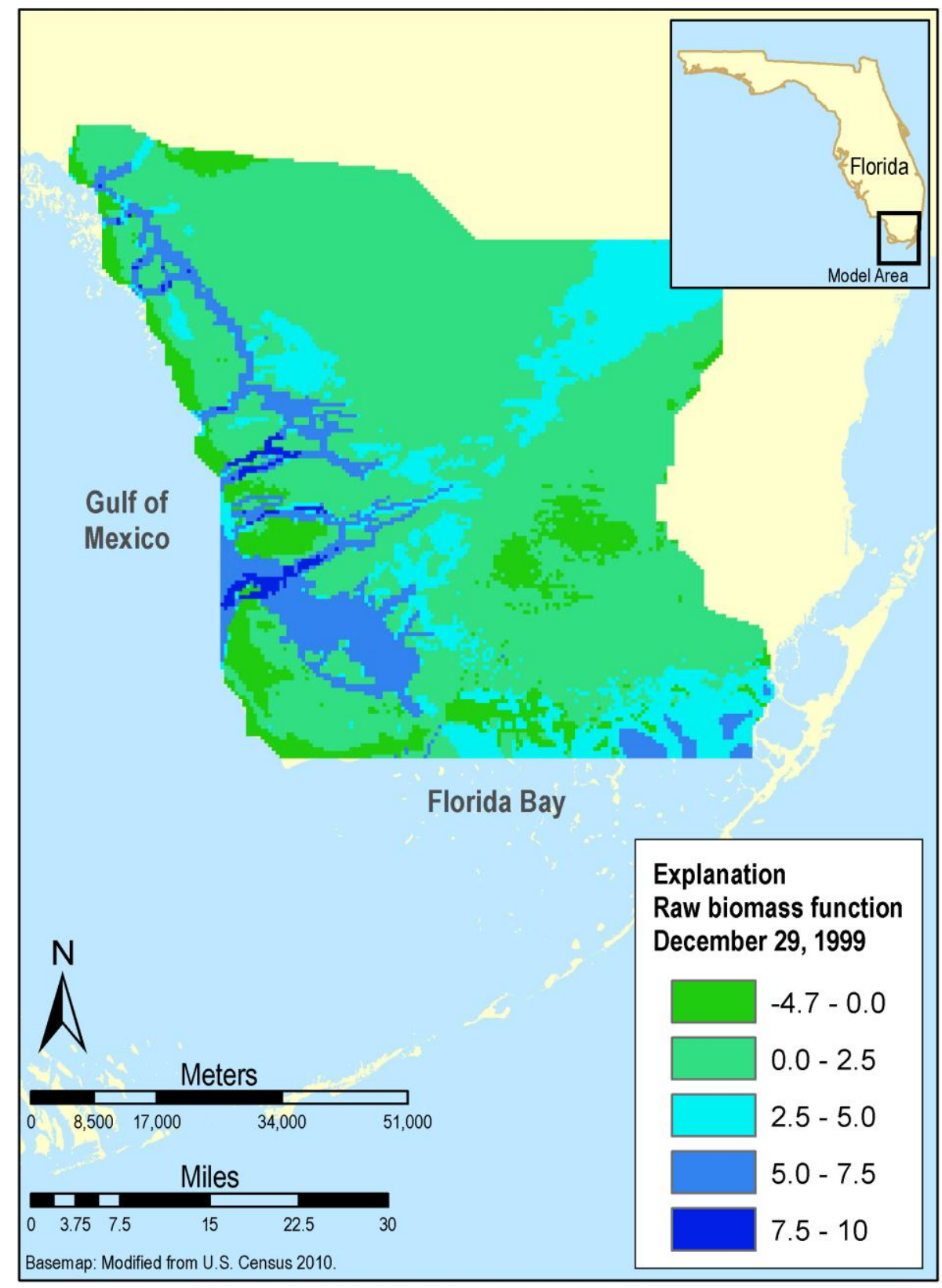

Figure 8. Map showing Estuarine Prey Fish Biomass Availability model output from the raw biomass function on December 29, 1999. Blue indicates greater biomass and green indicates lower biomass.

Raw Biomass-This measure is a function of the water depth input and the 90-day low salinity day count, mean 300-day depth, 90-day depth standard deviation, 60-day low depth day count, and continuous high depth day count outputs. A single map is produced for each day after the 299th day of the water depth time series (fig. 8). This output is given by equation 1; each parameter is the output or input for the day for which the raw biomass is being calculated, where all additions and multiplications are performed cell-wise. 

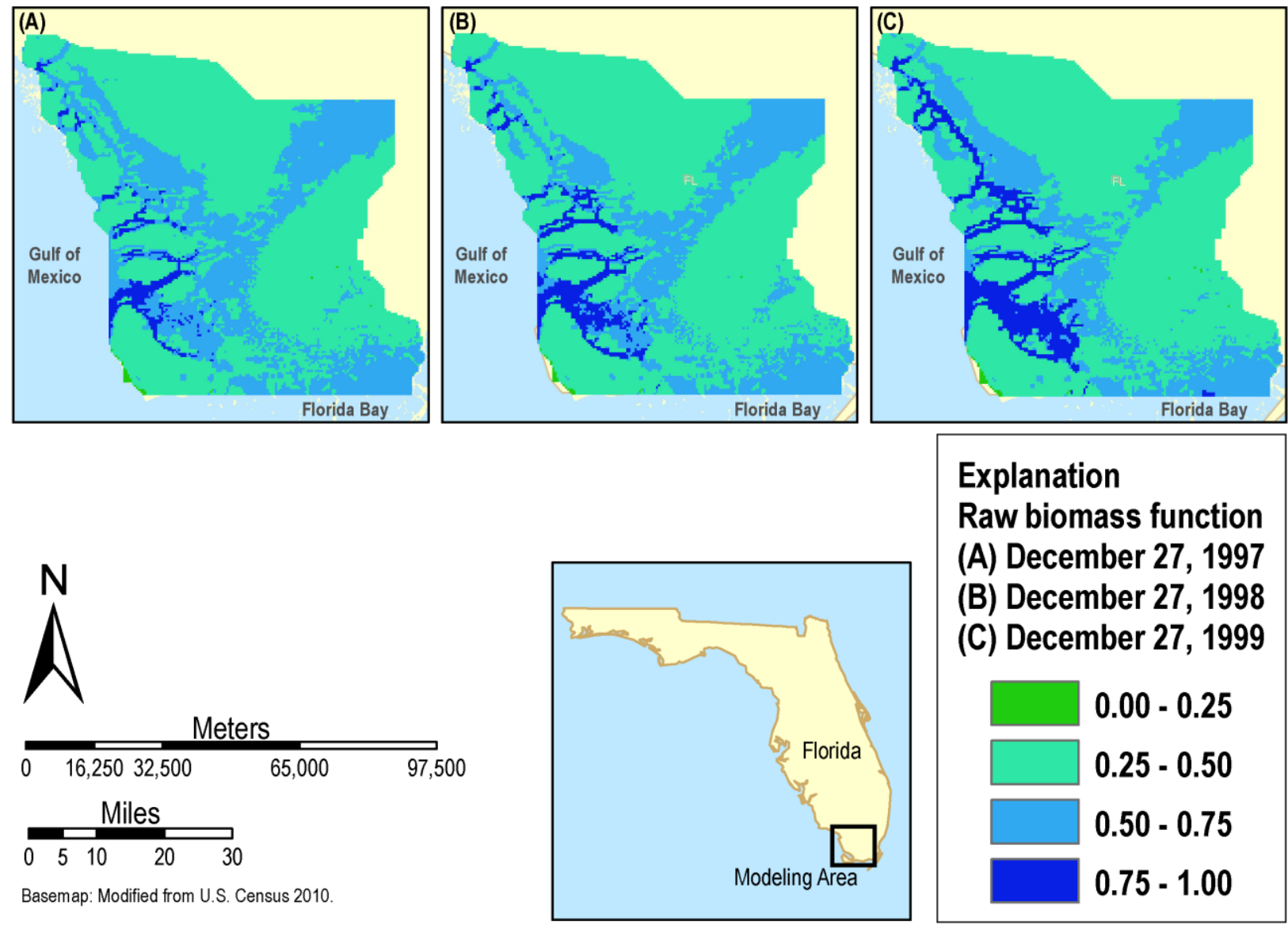

\section{Explanation}

Raw biomass function

(A) December 27, 1997

(B) December 27, 1998

(C) December 27, 1999

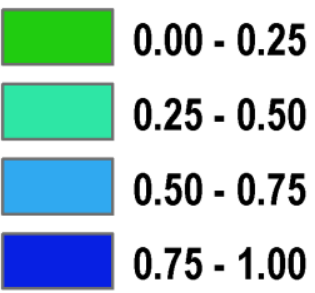

Figure 9. Map showing Estuarine Prey Fish Biomass Availability model output from the biomass index showing change through time on December 27, A, 1997, B, 1998, and C, 1999. Blue indicates greater biomass index value and green indicates lower biomass index value.

Biomass Index-This index is a function of the raw biomass output. A single map is produced for each day of the raw biomass (fig. 9). Each cell of the biomass index is calculated as per equation 2:

$$
\frac{(r-n)}{(m-n)}
$$

where $r$ is the cell in the raw biomass, $n$ is the minimum raw biomass value in all cells for all days, and $m$ is the maximum raw biomass value in all cells for all days. 


\section{Model Application User's Guide}

Requirements-The Estuarine Prey Fish Biomass Model application (biomass model) is written in Java using version 1.6.0_05 of the Java libraries. Consequently, version 1.6.0_05 or later of the Java Runtime Environment must be installed to run the application, which uses version 4.0 of the Java NetCDF libraries - these are included in the executable archive for convenience.

The program uses relatively little memory, at least 1 gigabyte (GB) of unallocated system memory for the application's use is required to ensure ample memory is available and to minimize the chance of running out of memory. The program should work on any processor that supports the Java Runtime Environment, although faster processor speeds result in shorter run times. For most data sets, it would be fairly reasonable to expect that the application will generate roughly $1 \mathrm{~GB}$ of output data, so there should be at least $1 \mathrm{~GB}$ of free hard drive space available on the drive containing the output directory for each year of data to be processed. For larger data sets, more space will be required for each year.

Usage-The biomass model application is packaged as a compressed zip" file. To run the application, the user must first extract all files locally and then double-click the JEMPreyBiomassModel.exe file. The model interface will appear, allowing the user to load a preexisting model parameter settings $\mathrm{xml}$ file or to manually set the model variables, including input and output folder paths.

Settings-The settings file is an extensible markup language (XML) file containing specific model parameters that can load the application with preset values for model variables, constants, and paths. The application allows the user to save model variable and path modifications as new settings files, or to load previously saved settings files.

Sample XML Settings File-The following is a sample XML settings file for a complete model run. The file, variable, and dimension names are just examples and may differ depending upon the input data used.

$<$ ?xml version="1.0" encoding="UTF-8" ?>

$<$ settings $>$

$<$ map type $=$ "netcdf/fixed2d" />

$<$ depth netcdflocation $=$ "C: Data $\backslash$ TIME_depth.nc" netcdfvariable="Depth"

netcdfxdimension=" $\mathrm{x} "$

netcdfydimension="y" netcdftimedimension="time" multiplier="100" />

$<$ salinity netcdflocation ="C:\Data\TIME_salinity.nc" netcdfvariable="Salinity"

netcdf $x$ dimension=" $x "$ netcdfydimension=" $y "$ netcdftimedimension="time" $>>$

$<$ output folder="C: $\backslash$ Outputs $\backslash$ PreyBiomassModel" />

$</$ settings $>$

Map Element-The root element of the settings XML file must contain an element named map." This element must have at least one attribute named type," which should be a string containing the type of data to be used in the model. Currently, the only type supported by the model application is netcdf/fixed2d, which denotes that the input data are contained in fixed two-dimensional grids in NetCDF file format.

Depth Element-The root element of the XML file must contain an element named -depth," and this element must have at least five attributes. The depth element must have an attribute named netcdflocation," which should be a string with the location of a NetCDF file containing a variable that stores water depths in meters. The depth element must also have an attribute named netcdfvariable," which should be a string containing the name of the variable for water depth in the NetCDF file. The 
depth element must also have an attribute named -netcdfxdimension," which should be a string containing the name of the $\mathrm{x}$ dimension for the water-depth variable in the NetCDF file, an attribute named -netcdfydimension," which should be a string containing the name of the y dimension for the water-depth variable in the NetCDF file, and an attribute named -netcdftimedimension," which should be a string containing the name of the time dimension for the water-depth variable in the NetCDF file.

Salinity Element-The root element of the XML file must contain an element named -salinity." This element must have at least five attributes. The salinity element must have an attribute named fetcdflocation," which should be a string with the location of a NetCDF file containing a salinity variable containing cells with values in parts per thousand. The salinity element must also have an attribute named -netcdfvariable," which should be a string containing the name of the variable for salinity in the NetCDF file. The salinity element must also have an attribute named netcdfxdimension," which should be a string containing the name of the $\mathrm{x}$ dimension for the salinity variable in the NetCDF file, an attribute named - netcdfydimension," which should be a string containing the name of the y dimension for the salinity variable in the NetCDF file, and an attribute named netcdftimedimension," which should be a string containing the name of the time dimension for the salinity variable in the NetCDF file.

Multiplier Attribute-The Depth and Salinity elements of the XML file can optionally contain an attribute named -multiplier," which should be a string containing a real number. If this attribute is set, then all cell values for the corresponding input (Depth or Salinity) will be multiplied by the multiplier before being processed.

Output element-The root element of the XML file must contain an element named - $\rightarrow$ utput." This element must have an attribute named folder, which is a string containing the destination path of the NetCDF files to be created or overwritten where spatial model results are to be stored.

Output-The model application produces a separate NetCDF file in the location specified in the XML settings file for each model input and output described in the Model Requirements section.

\section{Model Specifications}

Application design-The model application was originally developed to generate model results for the TIME model hydrology and salinity data (Wang and others, 2007), but was constructed using a design that would allow for future extension of the model application to other hydrology and salinity data. The model application was built using an extensible software plug-in framework, allowing for future integration with various other data manipulation and viewer applications built using similar architecture.

Mesh class-The Mesh class is an abstraction of any set of data that can be represented as an ordered list of cells containing floating-point numbers. The orientation, arrangement, and shape of the data is irrelevant, with the provision that any two Mesh objects representing the same data set should order the list of cells in exactly the same way. Implementations of Mesh must supply a very minimal set of methods (although the Mesh class contains several useful methods for convenience that are already implemented). Those methods that support basic interaction with Mesh data are passed an index, which is the location of the cell in the ordered list. The getCell method returns the floating point value contained in the cell at the location specified by the passed index. The setCell method takes a floating point value and assigns it to the cell at the location specified by the passed index. The getLength method returns the number of cells contained in the Mesh. The getCellCentroid returns the location of the centroid of the cell in the geographic coordinate system of the data. Finally, the clone method returns an exact duplicate of the Mesh. All of the model decision rules can be implemented upon any sort of data that can be represented as a Mesh using these five methods. 
MeshReader and MeshWriter interfaces-Because a function that maps the underlying data to a list of cells depends upon the nature of the data, a Mesh cannot be created directly. Likewise, because it is impossible to know the actual shape and arrangement of the underlying data using the five previously described methods, a Mesh cannot be stored directly in some medium, such as by being recorded to disk. To solve these problems, the MeshReader and MeshWriter interfaces are provided for the loading and saving of Mesh data, respectively.

An implementation of the MeshReader interface only needs to implement a single method named toad." The load method is passed a GregorianCalendar that contains the date of the data to be retrieved (which may be null if the data to be fetched has no time component); a String containing the name of the data to be fetched; and an array of Objects that contain any additional data required to populate the Mesh, which is returned by the method. The meaning of the additional data passed to the method is internal to the particular implementation of the MeshReader and does not necessarily have any external significance.

In like manner, an implementation of the MeshWriter interface only needs to implement a single method named -save." In this case, the save method is passed the Mesh to be stored; a GregorianCalendar that contains the date of the data to be stored (which may be null if the data to be stored has no time component); a String containing the name of the data to be stored; and an array of Objects that contain any additional data required to store the Mesh. As with the load method, the meaning of the additional data passed to the save method is internal to the particular implementation of the MeshWriter, and does not necessarily have any external significance.

Processor class-The Processor class is the component that implements the model decision rules. It is given a series of MeshReaders, as well as the other data needed to invoke the load method, for each of the various model inputs and one to load the saved outputs, a MeshWriter to store the model outputs, the time step dates for which the model is to be run, and the other parameters needed to generate the model results. The Processor class deals solely with Mesh objects, and does not interact with the underlying data in any way, which is what allows the Processor class to be used with any data set that can be converted into a Mesh object. The Processor class is responsible solely for generating model results. Inputs were previously verified to be in the correct format by the BiomassModel class.

BiomassModel class-The BiomassModel class gathers and tests the data necessary for the Processor class to generate model results. In order to meaningfully do this, the BiomassModel interacts with descendants of Mesh objects as their subtype, not abstractly. The precise restrictions and requirements imposed upon the input data by the BiomassModel class depend upon the input data, and are described fully in the applicable section on input data herein.

Current implementation: fixed 2D arrays-The Prey Biomass Model application is written to generate results for fixed two-dimensional regular rectilinear float arrays in NetCDF files with a daily time resolution. There are two inputs to the model application: water depth and salinity. 
Map requirements and restrictions-Both of the inputs contain map data. The water depth and salinity inputs both have the following requirements and restrictions:

- A daily time resolution

- At least one time step of data

- At least one cell in every map

- Identical cell dimensions

- Identical cell sets for all time steps and inputs, although the cell values may be different

- Contiguous time steps (that is, there must be a time step for every day from the beginning to the end of the range of time steps)

- All inputs must share at least one time step

- All data for a single input must be contained in a variable in a single NetCDF file in which the variable has three dimensions, one of which must be time, and contains floats

- All data must be in the same projection

Fixed 2D array implementation classes-A number of classes were developed to handle the reading of fixed two-dimensional grid data from two- and three-dimensional NetCDF variables, the writing of fixed two-dimensional grid data to two- and three-dimensional NetCDF variables, and the storage of fixed two-dimensional grid data in memory-all within the context of the Mesh architecture.

Fixed2Dgrid class-The Fixed2DGrid class is a simple implementation of the Mesh class intended to store regular, rectilinear, fixed two-dimensional grids. The size and dimensions of a Fixed2DGrid may not be changed once it has been created, but the values contained in the cells can change. This class has no exposed methods other than what is required by its Mesh superclass, and has no exposed constructor.

Fixed2DGridNetCDFReader class-The Fixed2DGridNetCDFReader class is an implementation of the MeshReader class intended to read regular, rectilinear, fixed two-dimensional grids from two- and three-dimensional NetCDF variables. When a Fixed2DGridNetCDFReader is instantiated, it must be passed a valid NetcdfFile object from which it will read data. The load method that is implemented from MeshReader returns a Fixed2DGrid from the passed NetcdfFile object. The GregorianCalendar object passed to the load method is the date of the Fixed2DGrid to be fetched from the NetCDF file (or null if the NetCDF variable has no time dimension). The String passed to the load method contains the name of the variable in the NetCDF file containing the data array to access. The additional parameters passed to the load method must contain two to three String objects. The first object must be a String containing the name of the $\mathrm{x}$ (easting) dimension of the NetCDF variable, and the second must be a String containing the name of the $y$ (northing) dimension of the variable. The third object must be a String containing the name of the time dimension of the NetCDF variable if the passed Gregorian Calendar is non-null (and is ignored otherwise).

Fixed2DGridNetCDFWriter class-The Fixed2DGridNetCDFWriter class is an implementation of the MeshWriter class intended to write Fixed2DGrids to two- and three-dimensional NetCDF variables. When a Fixed2DGridNetCDFWriter is instantiated, it must be passed a valid NetcdfFileWriteable object to which it will write data. The save method that is implemented from MeshWriter takes a Mesh object as a parameter, which must be the Fixed2DGrid that is to be stored in the NetCDF file. The GregorianCalendar object passed to the save method is the date of the Fixed2DGrid to be stored in the NetCDF file (or null if the NetCDF variable has no time dimension). The String passed to the save method contains the name of the variable in the NetCDF file to which the Fixed2DGrid is to be written. The additional parameters passed to the save method are the same as those passed to the load method, and must contain two to three String objects. The first object must be a String containing the name of the $\mathrm{x}$ (easting) dimension of the NetCDF variable, and the second must be 
a String containing the name of the y (northing) dimension of the variable. The third object must be a String containing the name of the time dimension of the NetCDF variable if the passed GregorianCalendar is non-null (and is ignored otherwise).

Outputs-The model application produces a separate NetCDF output file for each model input and output described in the Model Requirements section. The model results will contain the same range of time steps as the input files.

NetCDF files-The CF-1.0 compliant NetCDF output files of the model application contain variables for model inputs and outputs, as well as some variables that represent intermediary values in calculations and metadata. Variables in the output NetCDF files correspond to inputs and outputs described in the Model Requirements section where (1) NetCDF Variable Name is the name of the variable in its corresponding NetCDF output file, (2) Model Requirements Name is the name of the output in the Model Requirements section, (3) Units is the units of the values in the cells of the outputs, and (4) Type indicates whether the output is an input or output in the Model Requirements section (table 2).

Table 2. Variables in the output NetCDF files as they relate to inputs and outputs for the Estuarine Prey Fish Biomass Availability model.

\begin{tabular}{llll}
\hline \multicolumn{1}{c}{ NetCDF variable name } & \multicolumn{1}{c}{ Model requirements name } & \multicolumn{1}{c}{ Units } & \multicolumn{1}{c}{ Type } \\
\hline Water_Depth & Water Depth & Centimeters & Input \\
Salinity & Salinity & Parts per thousand & Input \\
Countinuous_High_Depth_Day_Count & Continuous High Depth Day Count & None (index) & Output \\
60-Day_Low_Depth_Day_Count & 60-Day Low Depth Day Count & None (index) & Output \\
90-Day_Low_Salinity_Day_Count & 90-Day Low Salinity Day Count & None (index) & Output \\
90-Day_Depth_Standard_Deviation & 90-Day Depth Standard Deviation & None (index) & Output \\
Mean_300-Day_Depth & Mean 300-Day Depth & None (index) & Output \\
Raw_Biomass & Raw Biomass & None (index) & Output \\
Biomass_Index & Biomass Index & None (index) & Output \\
\hline
\end{tabular}

In addition to the aforementioned variables, the NetCDF files contain a variable named transverse_mercator," which contains metadata to indicate that the map variables in the NetCDF files are in Universal Transverse Mercator (UTM) zone 17R. Output NetCDF files contain three dimensions: $\mathrm{x}, \mathrm{y}$, and time. Every variable for a map in the NetCDF file has the $\mathrm{x}$ and $\mathrm{y}$ dimensions. Variables for the maps in the NetCDF file that have a time component also have the time dimension.

Data set: TIME model-The model application was originally developed for the purpose of using TIME-model hydrology and salinity as inputs.

Input data sets: water depth and salinity-The water depth and salinity input data is from the TIME model hydrology and salinity (Wang and others, 2007). These water depth and salinity data are contained in a series of three-dimensional float arrays in a NetCDF file, and cover the time period from January 1, 1996, to December 30, 1999, with a daily resolution. The received data have a spatial extent covering the area from the coordinates $(461000,2779000)$ to $(557500,2865500)$ of UTM zone $17 \mathrm{R}$. Each cell of the received data is a square of 500 meters on a side, and the total area is 194 cells wide by 174 cells tall. The water depth data values contained in the cells are measured in meters and the salinity data values contained in the cells are measured in parts per thousand. 


\section{Future Use}

A number of potential changes may need to be made to the model application in the future for several reasons. This section addresses some of the most obvious foreseeable changes that may need to be made, and outlines the steps necessary to implement them.

Changing model rules-Although the model is currently in its final form with respect to the current model requirements, these requirements may change because of future discoveries or realizations by subject matter experts. In its current state, the model application allows for some flexibility with respect to changes to the model rules. If any of the weights or functions in the requirements are changed, then these may be modified directly in the configuration XML file. Any other changes to the model requirements would likely require modification or rewriting of the Processor class, and potentially the BiomassModel class as well.

Extending the model application to support new data sets-The model application was designed with the realization that other data sets would be used in the future to generate model results. The Mesh/MeshReader/MeshWriter and BiomassModel/Processor class hierarchies were developed in support of this anticipated need.

Known issues-There may be issues with NetCDF variables whose time units specify a time zone other than GMT, or variables that specify a time component other than 00:00, due to the functions of the DateUnit class in NetCDF libraries. This function returns dates parsed to the current time zone, not the time zone specified by the file. Consequently, loaded data may appear to be offset by a day, depending upon the time zone specified for the machine on which the application is run.

\section{Summary and Conclusions}

A comparison of model results to field data indicates that the model accurately predicts prey biomass in the estuarine regions of the model domain. Changes in the timing and distribution of freshwater deliveries across the Greater Everglades should result in increased water levels in areas occupied by prey fish in northeastern Florida Bay (Lorenz, 2000). Studies performed in the mangrove areas indicate that prey-base fish begin concentrating into deeper creeks and pools if wetland water levels fall below a depth threshold of $13.1 \mathrm{~cm}$. Out-of-season pulse releases resulting from upstream watermanagement activities rapidly raise water levels above the concentration threshold and fish disperse across the surface of the wetland. This eliminates the abundant and easily captured food resources needed by higher trophic-level species such as roseate spoonbills and American crocodiles. Even brief reversal events lasting 3 to 5 days can result in total failure of the fish prey base (Bjork and Powell, 1994). Projects proposed by CERP should alleviate this situation, leading to increased success of prey fishes, and in turn, an increase in the size of key species in the Greater Everglades.

\section{References Cited}

Bancroft, G.T., Strong, A.M., Sawicki, R.J., Hoffman, W., and Jewell, S.D., 1994, Relationships among wading bird forage patterns, colony locations, and hydrology in the Everglades, p. 615-658, in Davis, S.M., and Ogden, J.C., eds., Everglades: The Ecosystem and its restoration: Delray Beach, Fla., St. Lucie Press.

Bjork, R.D., and Powell, G.V.N., 1994, Relationships between hydrologic conditions and quality and quantity of foraging habitat for Roseate Spoonbills and other wading birds in the C-111 basin: Final report to the South Florida Research Center, Everglades National Park, Homestead, Fla. 
DeAngelis, D.L., Loftus, W.F., Trexler, J.C., and Ulanowicz, R.E., 1997, Modeling fish dynamics and effects of stress in a hydrologically pulsed ecosystem: Journal of Aquatic Ecosystem Stress and Recovery, v. 6, p. 1-13.

Frezza, P.E., and Lorenz, J.J., 2003, Distribution and abundance patterns of submerged aquatic vegetation in response to changing salinity in the mangrove ecotone of northeastern Florida Bay, in Florida Bay Program and Abstracts form the Joint Conference on the Science and Restoration of the Greater Everglades and Florida Bay Ecosystem: Gainesville, University of Florida.

Light, S.S., and Dineen, J.W., 1994, Water control in the Everglades: A historical perspective, in Davis S.M., and Ogden, J.C., eds, Everglades: The Ecosystem and Its Restoration: Delray Beach, Fla., St. Lucie Press.

Loftus, W.E, Eklund, A.M., 1994, Long-term dynamics of an Everglades small-fish assemblage, p. 461-484, in Davis S.M., and Ogden, J.C., eds, Everglades: The Ecosystem and its Restoration: Delray Beach, Fla., St. Lucie Press.

Lorenz, J.J., 1999, The response of fishes to physicochemical changes in the mangroves of northeast Florida Bay: Estuaries, v. 22, p. 500-517

Lorenz, J.J., 2000, Impacts of water management on Roseate Spoonbills and their piscine prey in the coastal wetlands of Florida Bay: Coral Gables, Fla., University of Miami, Ph.D. dissertation.

Lorenz, J.J., and Serafy, J.E., 2006, Subtropical wetland fish assemblages and changing Salinity regimes: Implications of Everglades restoration, Hydrobiologia, v. 569, p. 401-422.

McIvor, C.C., Ley, J.A., and Bjork, R.D., 1994, Changes in freshwater inflow from the Everglades to Florida Bay including effects on the biota and biotic processes: A review, in Davis, S.M., and Ogden, J.C., eds., Everglades: The Ecosystem and Its Restoration: Delray Beach, Fla., St. Lucie Press, p. 117146.

Montague, C.L., and Ley, J.A., 1993, A possible effect of salinity fluctuation on abundance of benthic vegetation and associated fauna in northeastern Florida Bay: Estuaries, v. 16, p. 703-717.

Ross, M.S., Meeder, J.F., Sah, J.P., Ruiz, P.L., and Telesnicki, G., 2000, The southeast saline Everglades revisited: A half-century of coastal vegetation change: Journal of Vegetation Science, $\mathrm{v}$. 11, p. 101-112.

U.S. Army Corps of Engineers and South Florida Water Management District, 1999, Central and Southern Florida Project Comprehensive Review Study final integrated feasibility report and programmatic environmental impact statement: Jacksonville, Fla., U.S. Army Corps of Engineers, Jacksonville District.

Wang, J.D., Swain, E.D., Wolfert, M.A., Langevin, C.D., James, D.E., and Telis, P.A., 2007, Application of FTLOADDS to simulate flow, salinity, and surface-water stage in the southern Everglades, Florida: U.S. Geological Survey Scientific Investigations Report 2007-5010, 112 p. 